\title{
Synthesis and Electrical Properties of $\mathrm{MoO}_{3}$
}

José Brito $^{1}$, Miriam Santos ${ }^{1}$, Audel Santos ${ }^{1}$, Veronica Gallegos ${ }^{1}$, Iza Ronquillo ${ }^{1}$, Ricardo Carbajal ${ }^{1}$ and F. Paraguay-Delgado ${ }^{2}$

${ }^{1}$ Universidad Tecnológica de Chihuahua Sur, Chihuahua, Chihuahua, Mexico, ${ }^{2}$ Centro de Investigacion en Materiales Avanzados (CIMAV), Chihuahua, Chihuahua, Mexico

\section{INTRODUCTION}

Orthorhombic phase of $\mathrm{MoO} 3$ were synthesized using microwave radiation. The synthesized micrometric particles were ground in a high energy mechanical mill (MM) for $45 \mathrm{~min}$, in order to obtain a nanostructured powder [1]. The crystal size, the lattice parameters and microstrain changes including the change of oxygen vacancy concentration were monitored by X-ray diffraction. The approximated values were obtained employing the Rietveld refinement of X-ray diffraction patterns. Tests of electrical properties were carried out in the orthorhombic phase with and without MM, it can be seen that the sample with MM presented a good response, reaching its maximum conductance at $120^{\circ} \mathrm{C}$ (a low temperature), while for the sample without MM, its conductance was zero. The electrical propietie is found to be dependent of oxygen concentration. There is a correlation between the particular size, the concentration of the oxygen vacancies, Band gap decreaseand and the increase of the conductance. The materials morphology and microstructure were characterize by electron microscopy techniques and the surface area and band gap was determine by Brunauer-Emmett-Teller method.

Synthesis

Morphology Changes

Statistical distribution particles size

STRUCTURAL ANALYSIS

Rietveld Refinement

The PD of Rx, show a widening of the peaks, in the samples with MM, indicating the presence of nanostructures and microdeformations. This is confirmed by Rietveld refinement (see Tables1).

There is a correlation between the particular size, the concentration of the oxygen vacancies, Band gap decreaseand and the increase of the conductance (see Fig. 4).

\section{CONCLUSION}

Orthorhombic phases of $\mathrm{MoO} 3$ was synthetized through a microwave heat treatment, by mechanical grinding of high energy, in short time of 45 minutes it was possible to obtain nanoparticles with an average size of $44 \mathrm{~nm}$. Using the Rietveld refinement method, PDs were refined, obtaining information on their structure such as network parameters, crystallite size, percentage of phases, the presence of microtensions and apparent oxygen vacancies. The sample with MM presented a good response, reaching its maximum conductance at $120^{\circ} \mathrm{C}$, while for the sample without MM, its conductance was zero. As a result of the presence of oxygen vacancies generated during the MM. 

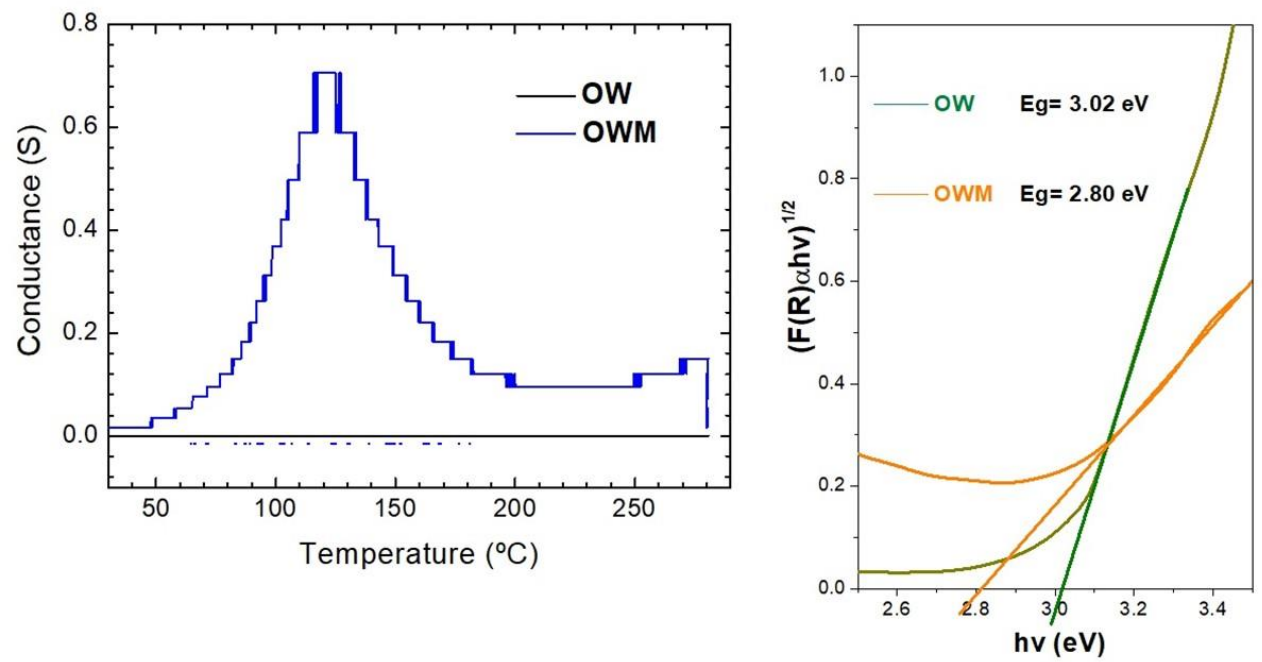

Figure 1. Fig.4 Changes originated after the MM a) .Conductance graph. b) Kubelka-Munk graph to calculate band energy interval

a)

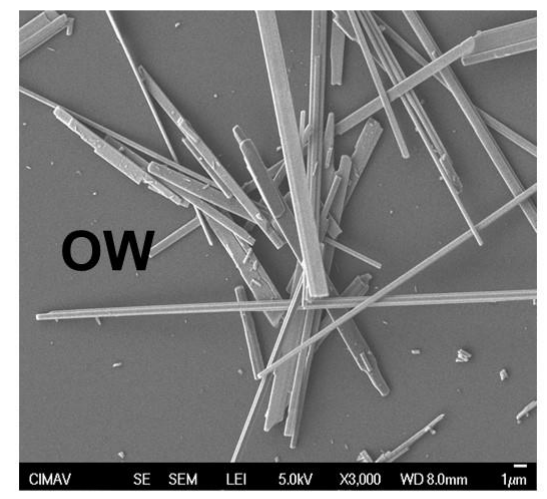

b)

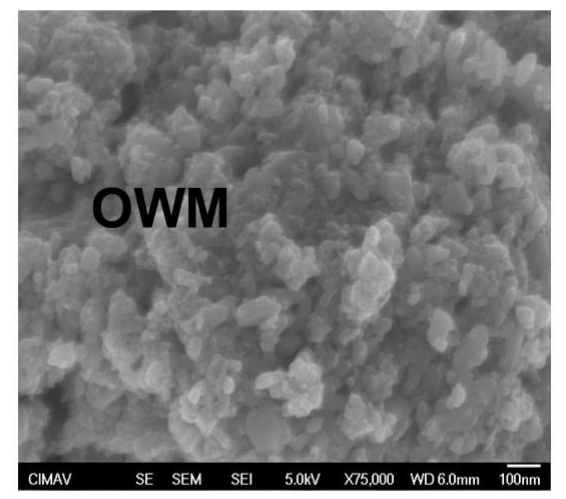

Figure 2. Fig. 1. a) The secondary electrons SEM image on OW simple, before milling, b) shows the SEM image of secondary electrons morphology was observed when the particles were subjected to mechanical milling (samples named OWM)

References

[1] M. Santos-Beltrán, F. Paraguay-Delgado, A. Santos-Beltrán, L. Fuentes, Journal of Alloys and Compounds, 648 (2015) 445-455.

[2] Khojier, H. Savaloni, S. Zolghadra Applied Surface Science, Volume 320, 30 November 2014, Pages 315-321.

[3] Arumugam Manivel, Gang-Juan Lee, Chin-Yi Chen, Jing-Heng Chen, Shih-Hsin Ma, Tzzy-Leng Horng d, Jerry J. Wu. Materials Research Bulletin 62 (2015) 184-191

[4] Praveen Meduri, Ezra Clark, Jeong H. Kim, Ethirajulu Dayalan, Gamini U. Sumanasekera and Mahendra K. Nano Lett. 2012, 12, 1784-1788 